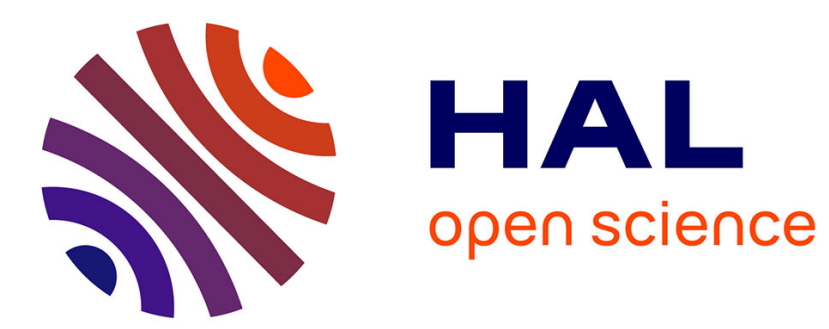

\title{
CANON À ÉMISSION DE CHAMP POUR MICROANALYSE PAR SPECTROSCOPIE D'ÉLECTRONS AUGER
}

P Morin, F. Simondet

\section{- To cite this version:}

P Morin, F. Simondet. CANON À ÉMISSION DE CHAMP POUR MICROANALYSE PAR SPECTROSCOPIE D'ÉLECTRONS AUGER. Journal de Physique Colloques, 1984, 45 (C2), pp.C2-307C2-308. 10.1051/jphyscol:1984268 . jpa-00223982

HAL Id: jpa-00223982 https://hal.science/jpa-00223982

Submitted on 1 Jan 1984

HAL is a multi-disciplinary open access archive for the deposit and dissemination of scientific research documents, whether they are published or not. The documents may come from teaching and research institutions in France or abroad, or from public or private research centers.
L'archive ouverte pluridisciplinaire HAL, est destinée au dépôt et à la diffusion de documents scientifiques de niveau recherche, publiés ou non, émanant des établissements d'enseignement et de recherche français ou étrangers, des laboratoires publics ou privés. 


\title{
CANON A ÉMISSION DE CHAMP POUR MICROANALYSE PAR SPECTROSCOPIE D'ÉLECTRONS AUGER
}

\author{
P. Morin et F. Simondet ${ }^{*}$ \\ Département de Physique des Matériaux, Université Claude Bernard, Lyon I, \\ 69622 Vilieurbanne Cedex, Erance \\ *ISA RIBER, 133-137 Boulevard National, 92503 Rueit-Malmaison, France
}

Résumé - Un canon à émission de champ destiné à la microanalyse Auger a été développé. La résolution spatiale de la sonde a été déterminée expérimentalement en fonction de l'intensité et de l'énergie du faisceau électronique. Les possibilités d'analyse de la microsonde ont été testées sur des îlots d'argent déposés sur une matrice de silicium.

\begin{abstract}
An electrostatic field emission microscope has been built to performe Auger analysis in small area $(0,1 \mathrm{~m})$ in the 1 to $10 \mathrm{keV}$ energy range. Micro Auger analysis have been demonstrated using silver clusters in a silicon matrix as a function of beam energy.
\end{abstract}

Les canons à émission de champ grâce à leur faisceau électronique de brillance élevée offrent de nouvelles possibilités en microanalyse Auger ; la résolution spatiale des analyses étant actuellement essentiellement limitée par les caractéristiques de la sonde, c'est à dire par le diamètre du faisceau pour une intensité et une énergie données.

La microsonde que nous avons développée est entièrement électrostatique. Le canon à émission de champ est constitué :

- d'une pointe de tungstène d'orientation [111],

- d'une lentille de type Enzel,

- d'un porte diaphragmes permettant la sélection de trois diaphragmes,

- d'un dispositif de correction de l'astigmatisme,

- d'un déflecteur électrostatique pour le balayage du faisceau.

Ce canon est monté sur une bride de $6^{\prime \prime}$ sa longueur est de $11 \mathrm{~cm}$. L'échantillon incliné à $45^{\circ}$ est situé à $5 \mathrm{~cm}$ de l'extrémité du canon. Un analyseur cylindrique à miroir est orienté perpendiculairement au faisceau électronique.

Pour résoudre le problème des fluctuations d'intensité du faisceau inhérentes à l'émission de champ, nous sommes intervenus à deux niveaux : d'une part en chauffant modérément la pointe au cours de l'émission et d'autre part en normalisant le signal Auger par un signal détecté sur un diaphragme. Le bruit résiduel correspondant mesuré sur les spectres Auger est ainsi toujours inférieur à $1 \%$ et le canon peut fonctionner une journée sans interruption.

Cet appareil est avant tout un microscope électronique à balayage permettant d'observer la surface des échantillons en détectant les électrons secondaires. Les observations Fig. 1 sont effectuées sur un échantillon de silicium présentant des bandes d'aluminium obtenues par lithographie par faisceaux d'électrons (échantillon fourni par M. Pantel et A. Georges du C.N.E.T. de Grenoble). Fig. la, les bandes d'aluminium sont espacées de $5 \mu m$ et leur largeur varie de $0,15 \mu \mathrm{m}$ à $1 \mathrm{\mu m}$. Fig. Ib la largeur des bandes est environ $0,1 \mu \mathrm{m}$. Ces observations montrent que la résolution est meilleure que $0,1 \mu \mathrm{m}$ avec un faisceau ayant une intensité de $3 \mathrm{nA}$ et une énergie de $10 \mathrm{keV}$.

Les caractéristiques résolution-intensité ont été tracées fig. 2 pour différentes énergies de faisceau. Ces courbes donnent la largeur à mi-hauteur de la distribution spaciale des électrons de la sonde. Cette largeur à mi-hauteur a été estimée par l'examen de petits détails observés sur des images obtenues en mode secondaire.

Les possibilités d'analyse de petites zones ont été testées sur des ilôts d'argent déposés sur un substrat de silicium. Un exemple de spectre $N(E)$ obtenu sur un ilôt d'argent de $0,1 \mu \mathrm{m}$ de diamètre est montré Fig. 3 pour un courant de sonde de $10 \mathrm{n} A$, et une énergie 
de faisceau de $10 \mathrm{keV}$ et une vitessse d'enregistrement de $1 \mathrm{eV} / \mathrm{s}$. Le rapport pic/fond continu de $1 / 5$ est plus faible que dans le cas d'un échantillon massif d'argent (voisin de 1).

Une proportion importante d'électrons secondaires sont créés dans la matrice de silicium par les électrons rétrodiffusés autour de l'ilôt d'argent. En diminuant l'énergie des électrons primaires, l'influence des rétrodiffusés est réduite, mais pour une optique électronique donnée, la taille de la sonde augmente, un compromis diamètre de sonde énergie de faisceau doit être trouvé. Des spectres similaires à celui de la figure 3 ont été obtenus avec des faisceaux de $5 \mathrm{keV}$ et de $3 \mathrm{keV}$ sur des ilôts de $0,2 \mu \mathrm{m}$ et de $0,3 \mu \mathrm{m}$ respectivement.

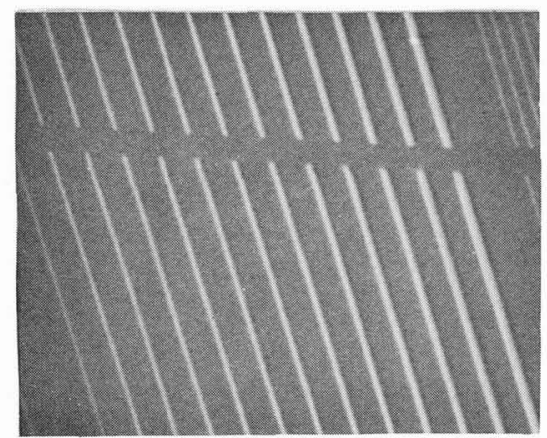

- a -
25 una.

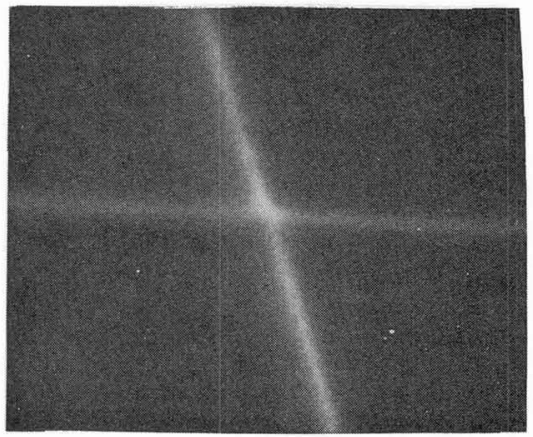

$-b-$

$0,2 \mu \mathrm{m}$

Figure 1: Aluminium sur silicium
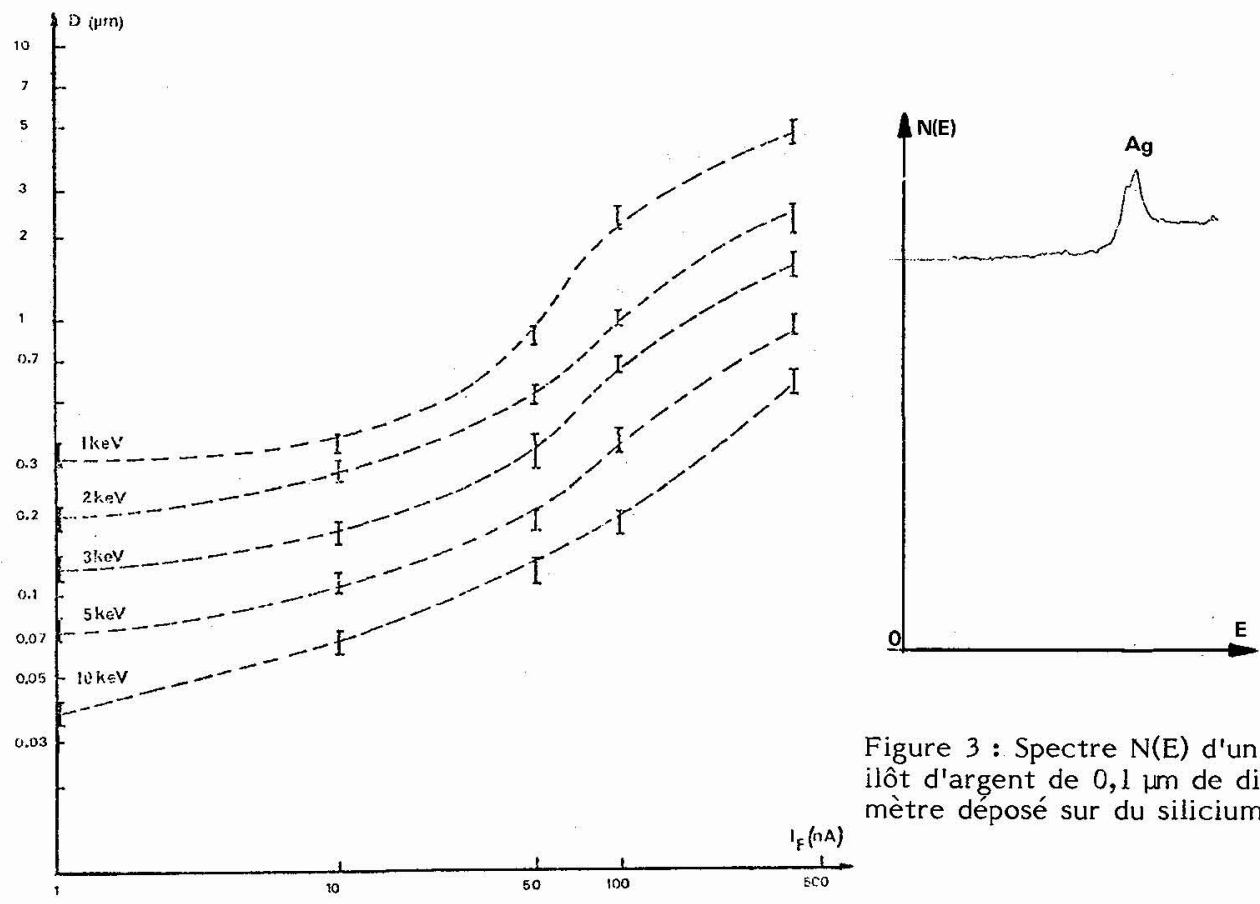

Figure 3 : Spectre $N(E)$ d'un ilôt d'argent de $0,1 \mu m$ de diamètre déposé sur du silicium.

Figure 2 : Diamètre de la sonde en fonction de l'intensité et de l'énergie du faisceau. 